\title{
A Note on the Existence of nonsmooth nonconvex Optimization Problems
}

\author{
Kazufumi Ito · Karl Kunisch
}

Received: date / Accepted: date

\begin{abstract}
Sufficient conditions for the existence of a solution to an abstract optimization problem in Banach spaces is given, which does not rely on convexity, regularity properties or a straightforward coerciveness assumption. Applications to sparsityconstrained optimization and to problems from mechanics are provided.
\end{abstract}

Keywords Nonsmooth, nonconvex optimization, coerciveness.

Mathematics Subject Classification (2000) 49J, 35 Q93.

\section{Introduction}

The existence of solutions to abstract minimization problems is typically based on assumption involving convexity properties and/or coercivity properties of the cost functional. This allows to consider minimizing sequences. Under appropriate compactness assumptions, depending on the fact whether the problem is posed in finiteor infinite dimensional spaces, and in case of the latter, whether the space is reflexive or not, a convergent subsequence can be extracted. Depending on closedness properties of the cost functional, accumulation points provide a desired minimizer for the optimization problem. The purpose of this note is to present a class of problems which does not allow to guarantee existence along this line of arguments, since the

Communicated by Giuseppe Buttazzo

K. Ito

Department of Mathematics, North Carolina State University, Raleigh, North Carolina, 27695-8205, USA; research partially supported by the Army Research Office under DAAD19-02-1-0394

E-mail: kito@ncsu.edu

K. Kunisch (corresponding author)

Institut für Mathematik, Karl-Franzens-Universität Graz, A-8010 Graz, Austria, supported in part by the Fonds zur Förderung der wissenschaftlichen Forschung under SFB 32, "Mathematical Optimization and Applications in the Biomedical Sciences"

E-mail: karl.kunisch@uni-graz.at 
cost is neither convex nor coercive. Examples are given to illustrate that such problems appear quite commonly in practice. The conditions that we provide guarantee existence of minimizers for these problems.

\section{The Existence Result and Applications}

We consider the problem

$$
\min _{x \in X} G(x)
$$

where $G$ is an extended, real-valued functional on a Banach space $X$. The mapping $G$ is not necessarily convex or smooth. There is no explicit assumption on $G$ to be bounded from below. We provide sufficient condition for $(\mathrm{P})$ to have a solution and give some inherently infinite dimensional examples to demonstrate their applicability.

Problem (P) has been adressed in several contributions before. In [2] the authors also consider the infinite dimensional case and provide examples from linear and nonlinear elasticity theory. Our condition is somewhat weaker than the condition in [2] and we provide different examples. In [1] the finite dimensional cases are studied in much details. In [3] necessary and sufficient conditions for existence to $(\mathrm{P})$ are obtained in terms of asymptotic behavior of $G$ along sequences, which are candidates for being minimizing sequences. While this is an elegant asymptotic analysis, for verifying existence in concrete applications the conditions given below are more direct and remain to be of independent importance.

Existence of solutions to $(\mathrm{P})$ will be obtained by considering the family of problems

$$
\min _{x \in X} G_{\varepsilon}(x)=G(x)+\frac{\varepsilon}{2}|x|^{2},
$$

for $\varepsilon$ tending to zero from above. While $G$ itself is not assumed to be coercive, this will be required for the functionals $G_{\varepsilon}, \varepsilon>0$. We first consider the case where $X$ is reflexive. The nonreflexive case will be addressed in Remark 2.1 (a) below. Recall that $G$ is called proper, iff its value is not equal to $\infty$ everywhere.

The term $\frac{\varepsilon}{2}|x|^{2}$ that is added to the cost here, serves the purpose that, together with other structural properties of the problem data, but without assumption on coercivity on $G$, existence of a solution to the original, unregularized problem (P) can be guaranteed. Once existence is obtained different, problem dependent, regularisation terms can be used to analyse properties, like regularity and stability, of the minimizers. This will not be the aim of this note.

Theorem 2.1 (Existence) Let $G$ be an extended real-valued functional on a reflexive Banach space $X$ satisfying the following properties:

(i) $G$ is proper and weakly lower semi-continuous,

(ii) $G_{\varepsilon}$ is coercive, i.e. $G_{\varepsilon}(x) \rightarrow \infty$ for $|x| \rightarrow \infty$, for each $\varepsilon>0$,

(iii) for any sequence $\left\{x_{n}\right\}$ in $X$ with $\left|x_{n}\right| \rightarrow \infty, \frac{x_{n}}{\left|x_{n}\right|} \rightarrow \bar{x}$ weakly in $X$, and $\left\{G\left(x_{n}\right)\right\}$ bounded from above, we have

(iiia) $\frac{x_{n}}{x_{n}} \rightarrow \bar{x}$ strongly in $X$, and 
(iiib) there exist $\left.\rho_{n} \in\right] 0,\left|x_{n}\right|\left[\right.$ and $n_{0}=n_{0}\left(\left\{\rho_{n}\right\},\left\{x_{n}\right\}\right)$ such that $G\left(x_{n}-\rho_{n} \bar{x}\right) \leq G\left(x_{n}\right)$, for all $n \geq n_{0}$.

Then $(\mathrm{P})$ admits a global solution.

Proof Let $\left\{\varepsilon_{n}\right\}$ be a sequence satisfying $\varepsilon_{n} \downarrow 0$. For $n$ fixed, let $\left\{x_{k}\right\}$ be a minimizing sequence for $\left(P_{\varepsilon_{n}}\right)$. By $(i i)$ this sequence is bounded. Extracting a weakly convergent subsequence from $\left\{x_{k}\right\}$ and using $(i)$, existence of a solution $x_{n} \in X$ for $\left(P_{\varepsilon_{n}}\right)$ can be argued in a standard manner.

Below we shall prove that $\left\{x_{n}\right\}$ is bounded. Then, there exists a weakly convergent subsequence, denoted by the same symbols, and $x^{*}$ such that $x_{n} \rightarrow x^{*}$. Passing to the limit, as $\varepsilon_{n} \downarrow 0$, in

$$
G\left(x_{n}\right)+\frac{\varepsilon_{n}}{2}\left|x_{n}\right|^{2} \leq G(x)+\frac{\varepsilon_{n}}{2}|x|^{2} \text { for all } x \in X
$$

and using again (i) we have

$$
G\left(x^{*}\right) \leq G(x) \text { for all } x \in X
$$

and thus $x^{*}$ is a minimizer for $G$.

We now argue that $\left\{x_{n}\right\}$ is bounded, and assume to the contrary that $\lim _{n \rightarrow \infty}\left|x_{n}\right|=\infty$. Then, a weakly convergent subsequence can be extracted from $\frac{x_{n}}{\left|x_{n}\right|}$ such that, again dropping indices, $\frac{x_{n}}{\left|x_{n}\right|} \rightarrow \bar{x}$, for some $\bar{x} \in X$. Moreover by (iiia)

$$
\frac{x_{n}}{\left|x_{n}\right|} \rightarrow \bar{x} \text { strongly. }
$$

Choose $\rho_{n}>0$ and $k_{0}$ according to (iiib). Then for all $n \geq n_{0}$

$$
G\left(x_{n}\right)+\frac{\varepsilon}{2}\left|x_{n}\right|^{2} \leq G\left(x_{n}-\rho_{n} \bar{x}\right)+\frac{\varepsilon}{2}\left|x_{n}-\rho_{n} \bar{x}\right|^{2} \leq G\left(x_{n}\right)+\frac{\varepsilon}{2}\left|x_{n}-\rho_{n} \bar{x}\right|^{2} .
$$

It follows that

$$
\left|x_{n}\right| \leq\left|x_{n}-\rho_{n} \bar{x}\right|=\left|x_{n}-\rho_{n} \frac{x_{n}}{\left|x_{n}\right|}+\rho_{n}\left(\frac{x_{n}}{\left|x_{n}\right|}-\bar{x}\right)\right| \leq\left|x_{n}\right|\left(1-\frac{\rho_{n}}{\left|x_{n}\right|}\right)+\rho_{n}\left|\frac{x_{n}}{\left|x_{n}\right|}-\bar{x}\right|
$$

This implies that

$$
1 \leq\left|\frac{x_{n}}{\left|x_{n}\right|}-\bar{x}\right|
$$

which contradicts to (2..1), and concludes the proof.

In the literature, sufficient conditions for existence for $(\mathrm{P})$ are typically expressed in term of the recession functional $G_{\infty}$ associated to $G$. This is the extended real-valued functional defined by

$$
G_{\infty}(x):=\inf _{x_{n} \rightarrow x, t_{n} \rightarrow \infty} \liminf _{n \rightarrow \infty} \frac{1}{t_{n}} G\left(t_{n} x_{n}\right) .
$$

The condition that $G_{\infty} \geq 0$ is used in $[1,2]$. 
Corollary 2.1 The conclusion of the Theorem 2.1 remains correct, iff (ii) is replaced by

(ii') $G_{\infty}(x)>-\infty$ for each $x$.

It remains also valid, iff (iiib) is replaced by

(iiib') $G_{\infty}(x) \leq 0$ implies that $|x| \neq 1$.

Proof Condition (ii) was used to guarantee boundedness of minimizing sequences for $\left(P_{\varepsilon_{n}}\right)$. Suppose that $\left(i i^{\prime}\right)$ holds and that $\left\{x_{k}\right\}$ be a minimizing sequence for $\left(P_{\varepsilon_{n}}\right)$ which is not bounded. Then there exists a subsequence for which $\lim _{l \rightarrow \infty}\left|x_{k_{l}}\right|=\infty$. We have the estimate

$$
\begin{aligned}
\infty=\lim _{l \rightarrow \infty} \frac{\varepsilon_{n}}{2}\left|x_{k_{l}}\right| & \leq \liminf _{l \rightarrow \infty} \frac{1}{\left|x_{k_{l}}\right|} G\left(\left|x_{k_{l}}\right| \frac{x_{k_{l}}}{\left|x_{k_{l}}\right|}\right)+\lim _{l \rightarrow \infty} \frac{\varepsilon_{n}}{2}\left|x_{k_{l}}\right| \\
& \leq \liminf _{l \rightarrow \infty} \frac{1}{\left|x_{k_{l}}\right|}\left(G\left(x_{k_{l}}\right)+\frac{\varepsilon_{n}}{2}\left|x_{k_{l}}\right|^{2}\right) \leq 0,
\end{aligned}
$$

which gives a contradiction, and hence $\left\{x_{k}\right\}$ is bounded. Turning to the second claim let $\left\{x_{n}\right\}$ be such that $G\left(x_{n}\right)$ is bounded from above and $\frac{x_{n}}{\left|x_{n}\right|} \rightarrow \bar{x}$ strongly. We find that

$$
G_{\infty}(\bar{x}) \leq \liminf _{n \rightarrow \infty} \frac{1}{\left|x_{n}\right|} G\left(\frac{x_{n}}{\left|x_{n}\right|}\left|x_{n}\right|\right)=\liminf _{n \rightarrow \infty} \frac{1}{\left|x_{n}\right|} G\left(x_{n}\right) \leq 0 .
$$

By $\left(i i i^{\prime}\right)$ therefore $|\bar{x}| \neq 1$, which gives a contradiction to strong convergence of $\frac{x_{n}}{\left|x_{n}\right|} \rightarrow \bar{x}$.

Remark 2.1 (a) The assumption that $X$ be reflexive can be replaced by assuming that it is the dual space of a separable space $\tilde{X}$, i.e. $X=\tilde{X}^{*}$, if simultaneously weak lower semicontinuity is replaced by weak* lower semicontinuity. In fact, in this case, the Banach-Alaoglu-Bourbaki theorem and the metrizability of the unit ball in the weak* topology of $\tilde{X}^{*}$ imply that bounded sequences in $X$ contain weakly* convergent subsequences. The proof can then be conduced as before. This situation applies in particular to the case when $X=L^{\infty}(\Omega)$ and $\tilde{X}=L^{1}(\Omega)$.

(b) In [2] it is assumed that $G_{\infty} \geq 0$ and

$$
G(x-\rho \bar{x}) \leq G(x),
$$

for all $x \in X$ and $G_{\infty}(\bar{x})=0$. Thus, in view of (2..2) condition (iiib) is weaker. In [1] we find a more general version of the second part of $(i i i b)$ : there exists a $\left.\rho_{n} \in\right] 0,\left|x_{n}\right|[$ and $z_{n} \in X$ such that for all $n$ sufficiently large

$$
G\left(x_{n}-\rho_{n} z_{n}\right) \leq G\left(x_{n}\right), \quad\left|z_{n}-z\right| \rightarrow 0 \text { and }|z-\bar{x}|<1 .
$$

Our proof can easily be adapted to this condition.

(c) Inspection of the proof shows that the quadratic penalty in the definition of $G_{\varepsilon}$ can be replaced by a general functional $\varphi(|x|)$, where $\varphi: \mathbb{R}^{+} \rightarrow \mathbb{R}^{+}$is coercive and strictly monotonically increasing.

(d) We have also proved that a sequence $\left\{x_{n}\right\}$, that minimizes the regularized problems $\left(P_{\varepsilon_{n}}\right)$ has a subsequence that converges weakly to a minimizer of $G(x)$. 


\section{Example 2.1 ( $\ell^{0}$ minimization)}

We consider the following minimization problem in the sequence space $X=\ell^{2}$ with sparsity constraints:

$$
\min _{x \in \ell^{2}} G(x)=\frac{1}{2}|A x-b|_{2}^{2}+\beta|x|_{0},
$$

where $A$ is a bounded linear operator in the $\ell^{2},|\cdot|_{2}$ denotes the norm in $\ell^{2}$, and

$$
|x|_{0}=\text { the number of nonzero elements of } x \in \ell^{2} \text {. }
$$

It is assumed the the nullspace $N(A)$ of $A$ is finite and that its range $R(A)$ is closed. It is straightforward to check that conditions (i) and (ii) are satisfied. To verify (iii) suppose that $\left|x_{n}\right|_{2} \rightarrow \infty, G\left(x_{n}\right)$ is bounded from above and $\frac{x^{n}}{\left|x^{n}\right|} \rightarrow z$ in $X$. First, we show that $z \in N(A)$ and $\frac{x^{n}}{\left|x^{n}\right|} \rightarrow z$. Since $\left\{G\left(x_{n}\right)\right\}$ is bounded from above, there exists $M$ such that

$$
G\left(x_{n}\right)=\frac{1}{2}\left|A x_{n}-b\right|_{2}^{2}+\beta\left|x_{n}\right|_{0} \leq M, \text { for all } n .
$$

Since $R(A)$ closed, by the closed range theorem implies every element in $X$ can be uniquely decomposed as $x_{n}=x_{n}^{1}+x_{n}^{2} \in R(A)+N(A)$.

Consequently, $0 \leq\left|A x_{n}^{1}\right|_{2}^{2}-2\left(b, A x_{n}^{1}\right)_{2}+|b|_{2}^{2} \leq M$ and the sequence $\left\{\left|A x_{n}^{1}\right|\right\}$ is bounded. This implies that

$$
0 \leq\left|A\left(\frac{x_{n}^{1}}{\left|x_{n}\right|_{2}}\right)\right|_{2}^{2}-2 \frac{1}{\left|x_{n}\right|_{2}}\left(A^{*} b, \frac{x_{n}}{\left|x_{n}\right|_{2}}\right)_{2}+\frac{|b|_{2}^{2}}{\left|x_{n}\right|_{2}^{2}} \rightarrow 0
$$

and consequently $A \frac{x_{n}^{1}}{\left|x_{n}\right|_{2}} \rightarrow 0$ in $X$. By the closed range theorem this implies that $\frac{x_{n}^{1}}{\left|x_{n}\right|_{2}} \rightarrow \bar{x}^{1}=0$ in $\ell^{2}$. Since $\frac{x_{n}^{2}}{\left|x_{n}\right|_{2}} \rightarrow \bar{x}^{2}$ and by assumption $\operatorname{dim} N(A)<\infty$ it follows that $\frac{x_{n}}{\left|x_{n}\right|_{2}} \rightarrow z=\bar{x}^{2}$ strongly in $\ell^{2}$ and thus (iiia) holds.

To verify (iiib) first note that $\left|x_{n}\right|_{0}=\left|\frac{x_{n}}{\left|x_{n}\right|_{2}}\right|_{0}$ and since $\left\{\left|x_{n}\right|_{0}\right\}$ is bounded it follows that $|z|_{0}<\infty$. Since $\bar{x} \in N(A)$ condition (iiib) will follow from

$$
\left|\left(x_{n}^{1}+x_{n}^{2}-\rho z\right)_{i}\right|_{0} \leq\left|\left(x_{n}^{1}+x_{n}^{2}\right)_{i}\right|_{0} \quad \text { for all } i,
$$

and for all $n$ sufficiently large. Only the coordinates for which $\left(x_{n}^{1}+x_{n}^{2}\right)_{i}=0$ with $z_{i} \neq 0$ require our attention. Since $|z|_{0}<\infty$ there exists $\tilde{i}$ such that $z_{i}=0$ for all $i>\tilde{i}$. For $i \in\{1, \ldots, \tilde{i}\}$ we define $\mathscr{I}_{i}=\left\{n:\left(x_{n}^{1}+x_{n}^{2}\right)_{i}=0, z_{i} \neq 0\right\}$. These sets are finite. In fact, if $\mathscr{I}_{i}$ is infinite for some $i \in\{1, \ldots, \tilde{i}\}$, then $\lim _{n \rightarrow \infty, n \in \mathscr{I}_{i}} \frac{1}{\left|x_{n}\right|_{2}}\left(x_{n}^{1}+x_{n}^{2}\right)_{i}=0$. Since $\lim _{n \rightarrow \infty} \frac{1}{\left|x_{n}\right|_{2}}\left(x_{n}^{1}\right)_{i}=0$ this implies that $z_{i}=0$, which is a contradiction. Taking $\tilde{n}$ as the maximal index in $\left\{\mathscr{I}_{i}: i \in\{1, \ldots \tilde{i}\}\right\}$ we have $\left(x_{n}^{1}+x_{n}^{2}\right)_{i} \neq 0$ for all $i \in\{1, \ldots, \tilde{i}\}$ and $n \geq \tilde{n}$. Summarizing we showed that (2..5) holds for any $\rho>0$ and $n \geq \tilde{n}$, as desired, and thus (2..3) admits a solution.

\section{Example 2.2 (Obstacle problem)}

Consider

$$
\min \int_{\Omega}\left(\frac{1}{2}|\nabla u|^{2}-f u\right) d x \text { subject to } u(x) \leq \psi \text { for a.e. } x \in \Gamma \text {. }
$$


Here $\psi \in L^{1}(\Gamma), f \in L^{2}(\Omega), \Omega$ is a bounded domain in $\mathbb{R}^{n}$ with Lipschitzean boundary $\partial \Omega$, and $\Gamma$ is a codimension 1 manifold in $\Omega$ with the property that $u$ restricted to $\Gamma$ satisfies $u_{\Gamma} \in L^{1}(\Gamma)$ for each $u \in H^{1}(\Omega)$. In case $n=1$ and $\left.\Omega=\right] 0,1[$ the condition can be $u\left(\frac{1}{2}\right) \leq \psi$. Note that $u$ is not fixed in any part of the domain.

This problem can be cast in the framework of $(\mathrm{P})$ by defining

$$
G(u)=\int_{\Omega}\left(\frac{1}{2}|\nabla u|^{2}-f u\right) d x+I_{\left\{u_{\Gamma} \leq \psi\right\}}(u),
$$

where $I_{S}$ denotes the indicator function of the set $S$. We choose $X=H^{1}(\Omega)$ and assume that

$$
\int_{\Omega} f d x \geq 0
$$

Condition $(i)$ is clearly satisfied. For $u \in X$ we have the estimate

$$
G_{\varepsilon}(u) \geq \int_{\Omega}\left(\frac{1}{2}|\nabla u|^{2}-f u-\frac{1}{2}|u|^{2}\right) d x \geq \frac{\varepsilon}{4}|u|^{2}-\frac{C}{\varepsilon}|f|_{L^{2}}^{2},
$$

where the constant $C$ is independent of $u$ and results from the continuous injection of $H^{1}(\Omega)$ in $L^{2}(\Omega)$. This implies $(i i)$.

Since constant functions are in the nullspace of the gradient operator condition (iii) requires some attention. We decompose any function $u \in L^{2}(\Omega)$ as $u=u_{1}+u_{2}$ where $u_{2}=\frac{1}{|\Omega|} \int_{\Omega} u d x$ is the projection of $u$ onto the space of constant functions and $u_{1}$ lies in the orthogonal complement. Next choose $\left|u_{n}\right|_{X} \rightarrow \infty, v_{n}=\frac{u_{n}}{\left|u_{n}\right|_{X}} \rightarrow v$, with $\left\{G\left(u_{n}\right)\right\}$ be bounded from above. Then $I_{\left\{u_{\Gamma} \leq \psi\right\}}\left(u_{n}\right)=0$ for all $n$ and

$$
\frac{1}{2} \int_{\Omega}|\nabla v|^{2} d x \leq \liminf _{n \rightarrow \infty} \frac{1}{2} \int_{\Omega}\left|\nabla v_{n}\right|^{2} d x \leq \limsup _{n \rightarrow \infty} \frac{1}{\left|u_{n}\right|_{X}} \int_{0}^{1} f v_{n} d x+\frac{G\left(u^{n}\right)}{\left|u_{n}\right|_{X}^{2}}=0 .
$$

Thus $\nabla v_{n} \rightarrow 0$ and $v_{n} \rightarrow v=v_{2}$ strongly for some constant $v_{2}$, which implies (iiia). Since $v_{n}(x) \leq \frac{\psi(x)}{\left|u_{n}\right|_{X}} \rightarrow 0$, we have $v_{2} \leq 0$ for a.e. $x \in \Gamma$. Consequently for any $\rho>0$

$$
G\left(u_{n}-\rho v\right)=G\left(u_{n}\right)+v_{2} \int_{0}^{1} \rho f(x) d x \leq G\left(u_{n}\right),
$$

where we use (2..7). Thus (iiib) holds for all $n$, and (2..6) has a minimizer.

\section{Example 2.3 (Friction problem)}

Consider

$$
\min _{u \in H^{1}(\Omega)} G(u)=\int_{\Omega}\left(\frac{1}{2}|\nabla u|^{2}-f u\right) d x+\int_{\Gamma}|u(s)| d s,
$$

where $\Omega$ and $f$ are as in Example 2 above and $\Gamma$ is part of the boundary, sufficiently regular such that the trace operation from $X=H^{1}(\Omega)$ to $L^{1}(\Gamma)$ is continuous. We assume that

$$
\left|\int_{\Omega} f d x\right|<|\Gamma|
$$


Turning to (iii) again we choose $\left\{u_{n}\right\}$ in $X$ such that $\left|u_{n}\right|_{X} \rightarrow \infty, v_{n}=\frac{u_{n}}{\left|u_{n}\right|_{X}} \rightarrow v$, for some $v$ in $X$, with $\left\{G\left(u_{n}\right)\right\}$ bounded. With $v_{n}=v_{n, 1}+v_{n, 2}$ we have

$$
\int_{\Omega}\left|\nabla v_{n, 1}\right|^{2} d x=\int_{\Omega}\left|\nabla v_{n}\right|^{2} d x \leq \frac{1}{\left|u_{n}\right|_{X}} \int_{\Omega} f v_{n} d x+\frac{1}{\left|u_{n}\right|_{X}} G\left(u_{n}\right) \rightarrow 0
$$

for $n \rightarrow \infty$. Hence $v_{n, 1} \rightarrow 0$ and $v_{n} \rightarrow v=v_{2}=\frac{1}{|\Omega|} \int_{\Omega} v d x$ strongly in $X$. For any $\rho_{n}>0$ we have

$$
G\left(u_{n}-\rho_{n} v\right)-G\left(u_{n}\right)=\rho_{n} v_{2} \int_{\Omega} f d x+\int_{\Gamma}\left(\left|u_{n}(s)-\rho_{n} v_{2}\right|-\left|u_{n}(s)\right|\right) d s .
$$

Now we choose $\rho_{n}=\frac{1}{2}\left|u_{n}\right|$ and obtain

$$
\lim _{n \rightarrow \infty} \frac{1}{\left|u_{n}\right|_{X}}\left(G\left(u_{n}-\rho_{n} v\right)-G\left(u_{n}\right)\right)=\frac{1}{2} v_{2} \int_{\Omega} f d x-\frac{1}{2}\left|v_{2}\right||\Gamma|<0,
$$

by (2..9). This implies that $\limsup _{n \rightarrow \infty} G\left(u_{n}-\rho_{n} v\right)-G\left(u_{n}\right) \leq 0$ and (iiib) follows.

\section{Example 2.4 ( $L^{\infty}$ Laplacian).}

To consider

$$
\min \int_{\Omega}|u-f| d x \text { subject to }|\nabla u(x)| \leq 1, \text { a.e. in } \Omega
$$

as a special case of the abstract result we set $X=H^{1}(\Omega)$ and

$$
G(u)=\int_{\Omega}|u-f| d x+I_{\{|\nabla u(\cdot)| \leq 1\}}(u)
$$

The set $\{u:|\nabla(u)| \leq 1\}$ is closed and convex in $X$, and hence weakly sequentially closed and (i) follows. Condition (ii) is clearly satisfied.

Next let $\left\{u_{n}\right\}$ in $H^{1}(\Omega)$ be such that $\left|u_{n}\right|_{X} \rightarrow \infty, v_{n}=\frac{u_{n}}{\left|u_{n}\right|_{X}} \rightarrow v$, for some $v$ in $H^{1}(\Omega)$, with $\left\{G\left(u_{n}\right)\right\}$ bounded. This implies that $\left|\nabla v_{n}\right|_{L^{2}} \leq \frac{1}{\left|u_{n}\right|_{X}} \rightarrow 0$ and $v_{n} \rightarrow v=v_{2}$ strongly, so that (iiia) holds. To verify (iiib) consider

$$
\frac{1}{\left|u_{n}\right|_{X}}\left(G\left(u_{n}-\rho_{n} v\right)-G\left(u_{n}\right)\right)=\int_{\Omega}\left|v_{n}-\frac{\rho_{n}}{\left|u_{n}\right|_{X}}-\frac{f}{\left|u_{n}\right|_{X}}\right| d x-\int_{\Omega}\left|v_{n}-\frac{f}{\left|u_{n}\right|_{X}}\right| d x .
$$

Choosing $\rho_{n}=\frac{1}{2}\left|u_{n}\right|_{X}$ we find

$$
\frac{1}{\left|u_{n}\right|_{X}}\left(G\left(u_{n}-\rho_{n} v\right)-G\left(u_{n}\right)\right) \rightarrow \frac{1}{2}\left|v_{2}\right||\Omega|-\left|v_{2}\right||\Omega|=-\frac{1}{2}\left|v_{2}\right||\Omega| .
$$

Hence $G\left(u_{n}-\rho_{n} v\right)-G\left(u_{n}\right) \leq 0$ for all $n$ sufficiently large, and hence (iiib) holds.

\section{Example 2.5 (Elastic contact problem)}

Consider the elastic contact problem for the deformation field $\mathbf{u} \in X=H^{1}(\Omega)^{2}$ and boundary body force $\mathbf{g}$ :

$\min \frac{1}{2} \int_{\Omega} \varepsilon(\mathbf{u}): \sigma(\mathbf{u}) d x+\int_{\Gamma_{1}} \mathbf{g} \cdot \mathbf{u} d s_{x}+\int_{\Gamma_{2}}|\tau \cdot \mathbf{u}| d s_{x}$, subject to $n \cdot \mathbf{u} \leq \psi$ on $\Gamma_{1} \cup \Gamma_{2}$, 
where we assume linear strain:

$$
\varepsilon(\mathbf{u})_{i, j}=\frac{1}{2}\left(\frac{\partial \mathbf{u}_{i}}{\partial x_{j}}+\frac{\partial \mathbf{u}_{j}}{\partial x_{i}}\right)
$$

and Hooke's law:

$$
\sigma=2 \mu \varepsilon+\lambda \operatorname{tr}(\varepsilon) I
$$

with positive Lame constants $\mu$ and $\lambda$. Here we assume the case $\Omega=] 0,1\left[^{2}\right.$ with $\Gamma_{1}=\left\{x_{2}=1\right\}$ (top) and $\Gamma_{2}=\left\{x_{2}=0\right\}$ (bottom), and $\mathbf{g}=\left(0, g_{2}\right)$ with $g_{2} \leq 0$ and $g_{2} \in L^{2}\left(\Gamma_{1}\right)$. We associate to this problem the mapping

$$
G(u)=\frac{1}{2} \int_{\Omega} \varepsilon(\mathbf{u}): \sigma(\mathbf{u}) d x+\int_{\Gamma_{1}} \mathbf{g} \cdot \mathbf{u} d s_{x}+\int_{\Gamma_{2}}|\tau \cdot \mathbf{u}| d s_{x}+I_{\left\{\left.n \cdot \mathbf{u}\right|_{\Gamma_{1} \cup \Gamma_{2}} \leq \psi\right\}}(\mathbf{u}) .
$$

Condition (i) is satisfied and (ii) follows from the Korn inequality. Turning to (iii) let $\left|\mathbf{u}_{n}\right|_{X} \rightarrow \infty \mathbf{v}_{n}=\frac{\mathbf{u}_{n}}{\left|\mathbf{u}_{n}\right|} \rightarrow v$, in $X$, with $\left\{G\left(\mathbf{u}_{n}\right)\right\}$ is bounded. We have

$$
\varepsilon\left(\mathbf{v}_{n}\right) \rightarrow 0
$$

This follows from

$$
0 \leq \frac{1}{2} \int_{\Omega} \varepsilon\left(\mathbf{v}_{n}\right): \sigma\left(\mathbf{v}_{n}\right) d x \leq \frac{G\left(\mathbf{u}_{n}\right)}{\left|\mathbf{u}_{n}\right|_{X}^{2}}-\frac{1}{\left|\mathbf{u}_{n}\right|_{X}} \int_{\Gamma_{1}} \mathbf{g \mathbf { v } _ { n }} d s_{x}-\frac{1}{\left|\mathbf{u}_{n}\right|_{X}} \int_{\Gamma_{2}}\left|\tau \cdot \mathbf{v}_{n}\right| d s_{X} \rightarrow 0
$$

for $n \rightarrow \infty$.

Therefore, as in the case of the obstacle problem we can decompose $v$ in an element of the kernel of $\varepsilon$, which is 0 , and another one in the orthogonal complement (w.r.t. $\left.L^{2}(\Omega)^{2}\right)$, given by $\left\{\left(-A x_{2}+C_{1}, A x_{1}+C_{2}\right): A \in \mathbb{R}, C_{1} \in \mathbb{R}, C_{2} \in \mathbb{R}\right\}$. Thus we have that $\mathbf{v}_{n} \rightarrow \mathbf{v}=\left(\mathbf{v}_{1}, \mathbf{v}_{2}\right) \in\left\{\left(-A x_{2}+C_{1}, A x_{1}+C_{2}\right)\right\}$ strongly in $X$, for constants $A, C_{1}, C_{2}$.

Further we find that $\mathbf{v}_{2}=\lim _{n \rightarrow \infty} \mathbf{v}_{2, n}=\lim _{n \rightarrow \infty} \frac{\mathbf{u}_{2, n}}{\left|\mathbf{u}_{n}\right|_{X}} \leq 0$ on $\Gamma_{1} \cup \Gamma_{2}$ and $\mathbf{v}_{1}=C_{1}$ on $\Gamma_{2}$.

Consequently with $\rho_{n}=\frac{1}{2}\left|u_{n}\right|_{X}$ we have

$$
\frac{G\left(\mathbf{u}_{n}-\rho \mathbf{v}\right)-G\left(\mathbf{u}_{n}\right)}{\left|\mathbf{u}_{n}\right|}=-\int_{\Gamma_{1}} \rho_{n} \mathbf{g}_{2} \mathbf{v}_{2} d s_{x}+\int_{\Gamma_{2}}\left(\left|\mathbf{v}_{n, 1}-\frac{1}{2} \mathbf{v}_{1}\right|-\left|\mathbf{v}_{n, 1}\right|\right) d s_{x}
$$

Since $\mathbf{g}_{2} \leq 0, \mathbf{v}_{2} \leq 0$ and $\mathbf{v}_{1}=C_{1}$ we obtain

$$
\limsup _{n \rightarrow \infty} \frac{G\left(\mathbf{u}_{n}-\rho_{n} \mathbf{v}\right)-G\left(\mathbf{u}_{n}\right)}{\left|\mathbf{u}_{n}\right|} \leq-\frac{1}{2}\left|\Gamma_{2}\right| C_{1}
$$

and hence $(i i i b)$ follows and (2..10) has a minimizer. 


\section{Conclusions}

A theorem providing sufficient conditions for nonsmooth, nonconvex optimization problems reflexive Banach spaces is given. Its applicability to $\ell^{0}$ optimization and to friction and contact problems is demonstrated. Generalization in several directions can be of interest. These include the study of regularization terms different from the quadratic one that was used in this paper. The introduction of additional explicit constraints is of relevance for applications. Applications to fracture mechanics, and to newly emerging nonsmooth nonconvex problems in mathematical image analysis should also be considered in future work.

\section{References}

1. A. Auslender and M. Teboulle, Asymptotic Cones and Functions in Optimization and Variational Inequalities, Springer, Heidelberg (2003)

2. C. Baiocchi, G. Buttazzo, F. Gastaldi, F. Tomarelli, General existence theorems for unilateral problems in continuum mechanics, Arch. Rational Mech. Anal., 100, 149-189 (1988)

3. J-P. Penot, Noncoercive problems and asymptotic conditions, Asymptotic Analysis, 49, 205-215 (2006) 